\title{
RR Lyrae stars in the Sagittarius dwarf galaxy: Period analysis ${ }^{\star}$
}

\author{
P. Cseresnjes ${ }^{1,2, \star \star}$ \\ 1 DASGAL, Observatoire de Paris, 61 avenue de l'Observatoire, 75014 Paris, France \\ 2 Centre d'Analyse des Images - INSU, France
}

Received 19 April 2001 / Accepted 1 June 2001

\begin{abstract}
We carried out a period analysis on $\sim 3700 \mathrm{RR}$ Lyrae stars on two Schmidt fields centred on $(l, b)=\left(3.1^{\circ},-7.1^{\circ}\right)$ and $\left(6.6^{\circ},-10.8^{\circ}\right)$ respectively, covering an area of $\sim 50 \mathrm{deg}^{2}$. These stars are distributed almost evenly between the Sagittarius dwarf galaxy (Sgr) and the Milky Way. For Sgr members, the average periods are $\left\langle P_{\mathrm{ab}}\right\rangle=0.574^{\mathrm{d}}$ and $\left\langle P_{\mathrm{c}}\right\rangle=0.322^{\mathrm{d}}$ for RRab and RRc stars respectively. This places Sgr in the long-period tail of the Oosterhoff I group. We report the detection of 53 double-mode RR Lyrae stars (RRd) within our sample. The magnitude of 40 of these stars is consistent with membership in Sgr whereas 13 RRds are located within our Galaxy. We also found 13 RR Lyraes (5 in Sgr and 8 in the Galaxy) exhibiting two closely spaced frequencies, most probably related to non-radial pulsations. The period distribution of the RR Lyrae variables in Sgr is compared to those of other Milky Way satellites. We find a remarkable similarity between the RR Lyrae populations in Sgr and the Large Magellanic Cloud (LMC), suggesting that these galaxies have similar horizontal branch morphologies. This may indicate that Sgr and the LMC started their formation under similar conditions. Using various photometric indicators, we estimate the metallicity of the RR Lyrae stars in Sgr and find $\langle[\mathrm{Fe} / \mathrm{H}]\rangle \simeq-1.6$ dex with a dispersion of $\sim \pm 0.5$ dex around this value and a minor but significant population at $\lesssim-2.0$ dex. We do not find evidence for a spatial metallicity gradient in the RR Lyrae population of Sgr. From the spatial distribution of RR Lyraes, we find that the main body of Sgr contains 4200 RRab stars. Assuming that population gradients are negligible in Sgr, we find $M_{V}(\mathrm{Sgr}) \simeq-13.9_{-0.6}^{+0.4} \mathrm{mag}$ for the main body. If Sgr has been stripped of $50 \%$ of its mass through Galactic tides, as assumed by some models, it would imply a total absolute magnitude of $\sim-14.7$ mag for this galaxy. Such a luminosity would be consistent with the empirical metallicity/luminosity relation for dwarf spheroidal galaxies ${ }^{\star \star \star}$.
\end{abstract}

Key words. stars: horizontal-branch - stars: population II - stars: variables: RR Lyr - galaxies: dwarf - galaxies: individual: Sagittarius dwarf - local group

\section{Introduction}

Since RR Lyrae stars are low mass helium core-burning stars, they are believed to be older than 10 Gyr. The study of the period distributions of these variables can therefore provide insights into the original populations of their host system. Field and cluster RR Lyraes show a clear separation between long period/metal poor and short period/metal rich stars, known as the Oosterhoff dichotomy (Oosterhoff 1939). On the other hand, the average period of RR Lyrae populations in Dsph galaxies is intermediate between the two Oosterhoff groups. It has

\footnotetext{
* Based on observations obtained at the European Southern Observatory, La Silla, Chile.

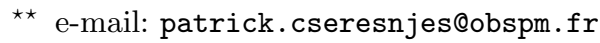

$\star \star \star$ Table 15 is only available in electronic form at the CDS via anonymous ftp to cdsarc.u-strasbg.fr (130.79.128.5) or via

http://cdsweb.u-strasbg.fr/cgi-bin/qcat?J/A+A/375/909
}

been shown that this intermediate status is not the consequence of a superposition of two populations but rather an intrinsic property of RR Lyrae populations in these systems (e.g. Sextans: Mateo et al. 1995; Leo II: Siegel \& Majewski 2000).

Of particular interest among RR Lyrae stars are those pulsating simultaneously in the fundamental and first overtone mode (RRd). These stars offer the opportunity to constrain their mass and luminosity independent of stellar evolution theory (e.g. Bono et al. 1996; Kovács \& Walker 1999). The exact status of these variables is still under debate. The intermediate position of RRd stars in the instability strip between RRc (pulsating in the first overtone mode) and RRab stars (pulsating in the fundamental mode) suggests that these stars are in the process of mode switching. This scenario seems however excluded by theoretical calculations (Cox et al. 1980) which yield a much too short duration for this transition state ( $\lesssim 10^{3-4}$ years) to account for the high fraction of $\mathrm{RRd}$ 
variables observed in some systems (e.g. IC 4499, M 68). It seems however that these stars are evolving rapidly (i.e. the changes are observable during a human life-time), and some period and amplitude changes have already been observed (Purdue et al. 1995; Clement et al. 1997; Paparó et al. 1998; Benkö \& Jurcsik 2000).

The first RRd star discovered was AQ Leo (Jerzykiewicz \& Wenzel 1977), a field RR Lyrae. After this discovery, searches were focused on old stellar systems harboring RR Lyrae stars and, surprisingly, RRd variables were searched successfully in some systems but vainly in others. RRd stars have been found in globular clusters (M 15: Nemec 1985; M 3: Nemec \& Clement 1989; NGC 2419 and NGC 2466: Clement \& Nemec 1990; M 68: Clement et al. 1993; IC 4499: Walker \& Nemec 1996), in Dsph galaxies (Draco: Nemec 1985; Sculptor: Kałużny et al. 1995) and in the Galactic Halo (Clement et al. 1991; GarciaMelendo \& Clement 1997; Clementini et al. 2000). On the other hand, searches for RRd variables were unsuccessful in $\omega$ Cen (Nemec et al. 1986), M 80, M 9 and NGC 2298 (Clement \& Walker 1991), Ursa Minor (Nemec et al. 1988) and 20 other globular clusters (Clement \& Nemec 1990). The parameter(s) driving the occurrence of RRd pulsators in stellar systems is still not clear and more observations are needed before any firm conclusion can be drawn. What is clear, however, is that the two Oosterhoff groups are well separated in a Petersen diagram (a plot of the period ratio versus the fundamental period - Petersen 1973), the OoI RRd pulsators having lower periods and period ratios than their OoII counterparts.

Recently, the collection of known RRd stars has been substantially increased with the discovery of 181 new RRd variables in the Large Magellanic Cloud (Alcock et al. 1997; Alcock et al. 2000b). These stars revealed a new picture because they were spread across the Petersen diagram, filling the gap between the two Oosterhoff groups. Pulsation models show that this distribution may be caused by a mass and/or metallicity spread within the population of RRd stars (Kovács 2000). Spectroscopic measurements on a sample of these stars seems to confirm the metallicity spread (Clementini et al. 2000; Bragaglia et al. 2001).

The search for multi-periodic RR Lyraes in the MACHO data set revealed other surprises. For instance, many RR Lyrae periodograms exhibited two closely spaced frequencies. This frequency pattern, first discovered by Olech et al. (1999a, 1999b) in RR Lyrae stars, cannot be explained by the superposition of radial pulsations and is therefore believed to be related to non-radial modes. Although these kind of stars have been detected in only four different places to date (M 55, M 5, LMC and Galactic Bulge), they seem to be relatively common in their host system. Theoretical modelling of these stars have been proposed by Van Hoolst et al. (1998) and by Dziembowski \& Cassisi (1999).

We carried out a period analysis on a sample of $\sim 3700$ RR Lyrae stars in Sgr and the Galactic Centre. The paper is structured as follows. Section 2 presents the

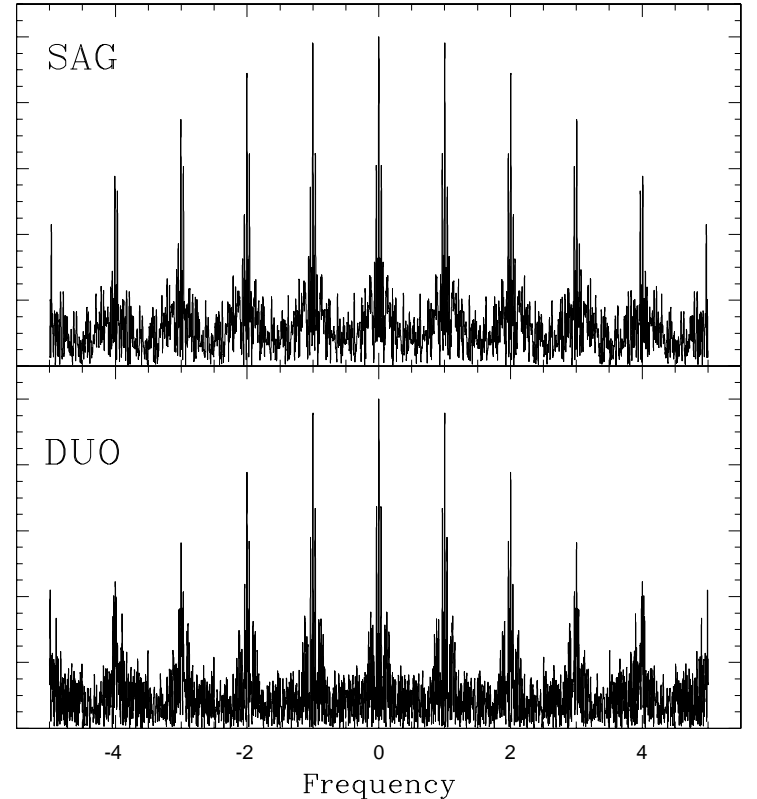

Fig. 1. Typical spectral windows for SAG (Upper panel) and DUO (lower panel).

data and their reduction. In Sect. 3, we describe the selection processes and present the sample of detected multiperiodic RR Lyraes. In Sect. 4, we present the period distributions of the RR Lyrae catalogs and compare them with those observed in other stellar systems. Section 5 is devoted to a presentation of the period-amplitude diagram of the RR Lyrae population in Sgr. In Sect. 6, we use photometric indicators to estimate the metallicity of the RR Lyrae population. Section 7 is devoted to a discussion about the spatial homogeneity of the RR Lyrae population and in Sect. 8 we estimate the RRab content of Sgr. Finally, we summarize our results and conclude in Sect. 9.

\section{Data}

\subsection{Observations and reduction}

The data discussed in this paper are based on two sets of photographic plates taken with the ESO $1 \mathrm{~m}$ Schmidt telescope at La Silla (Chile) between 1994 and 1996. The first set of observations consists of a series of $82 B_{\mathrm{J}}$ photographic plates centred on $(l, b)=\left(3.1^{\circ},-7.1^{\circ}\right)$ and taken between June 11th 1994 and October 7th 1994. The limiting magnitude of these plates reaches $B_{\mathrm{J}} \sim 20.5$. A first analysis of these data allowed the detection of microlensing events toward the Galactic bulge as part of the DUO project (Alard \& Guibert 1997). The second set of data results from a series of 68 Kodak Tech-Pan 4415 films combined with a BG12 filter centred on $(l, b)=\left(6.6^{\circ},-10.8^{\circ}\right)$. These observations spanned 83 days between May 17th and August 9th 1996. The exposure times resulted in a limiting magnitude of $V \sim 20$. Together with the first set of plates, this series allowed us to reveal the shape of the Sagittarius dwarf galaxy near the Galactic Plane 
(Alard 1996; Cseresnjes et al. 2000, hereafter Paper I). For convenience we will refer to the first field as the $D U O$ field whereas the second field will be called $S A G$ field. Typical spectral windows for $S A G$ and $D U O$ are shown in Fig. 1.

The plates were scanned at CAI/Paris Observatory with the high-speed microdensitometer MAMA ${ }^{1}$. The extraction of the sources were performed with the software Extractor written by Alard (Alard \& Guibert 1997). The final database contains the light curve for $\sim 20 \times 10^{6}$ stars.

\subsection{Photometry}

The $B_{\mathrm{J}}$ band is well known to astronomers and it suffices here to recall the photometric relation linking this band to the standard Johnson system $B_{\mathrm{J}}=B-0.28(B-V)$ (Blair \& Gilmore 1982). On the other hand, the emulsion used in the second field may not be familiar to the reader so we shall say some words about it. The TechPan 4415 emulsion is a fine grained, high resolution film with a sensitivity extending to $0.69 \mu \mathrm{m}$. Together with a BG12 filter it results in a band peaking at $\sim 0.39 \mu \mathrm{m}$ with a width of $\sim 0.15 \mu \mathrm{m}$ at half maximum transmission (see Fig. 1 in Paper I). We refer the interested reader to Phillipps \& Parker (1993), Parker \& Malin (1999) and the Kodak Web site ${ }^{2}$ for a more thorough description of the emulsion. The band resulting from the combination $4415+$ BG12 has been calibrated with a sequence of 1638 stars located within our field and which were published by Sarajedini \& Layden (1995). This calibration resulted in the relation $B_{4415}=V+1.47(V-I)$.

\section{Selection processes}

\subsection{Single-mode RR Lyraes}

The selection process for RR Lyrae stars is similar to the one used in Paper I, except that we adapted the parameters to allow detection of RRc stars. In short, we extracted from the data-base the variables with an amplitude $\gtrsim 0.2 \mathrm{mag}$. $\left(\chi^{2} / N_{\mathrm{DOF}}>5\right)$ and a minimum of 30 points in their light curve. These stars were then period searched between $0.2^{\mathrm{d}}$ and $10.0^{\mathrm{d}}$ using the multi-harmonic periodogram method of Schwarzenberg-Czerny (1996) and we fitted a Fourier series with up to five harmonics to the folded light curve. The variables with a well-defined light curve $\left(\sqrt{\chi 2 / \chi_{\text {fit }}^{2}}>1.5\right)$ were then plotted in the $R_{21} / \Phi_{21}$ plane where the RR Lyrae stars could easily be spotted. The final sample contains $\sim 3700$ RR Lyrae variables almost evenly distributed between Sgr and the Galaxy.

\footnotetext{
1 MAMA (http://dsmama.obspm.fr) is operated by INSU (Institut National des Sciences de l'Univers) and the Observatoire de Paris.

2 http://www.kodak.com/cluster/global/en/professional/ support/techPubs/p255/p255.shtml
}

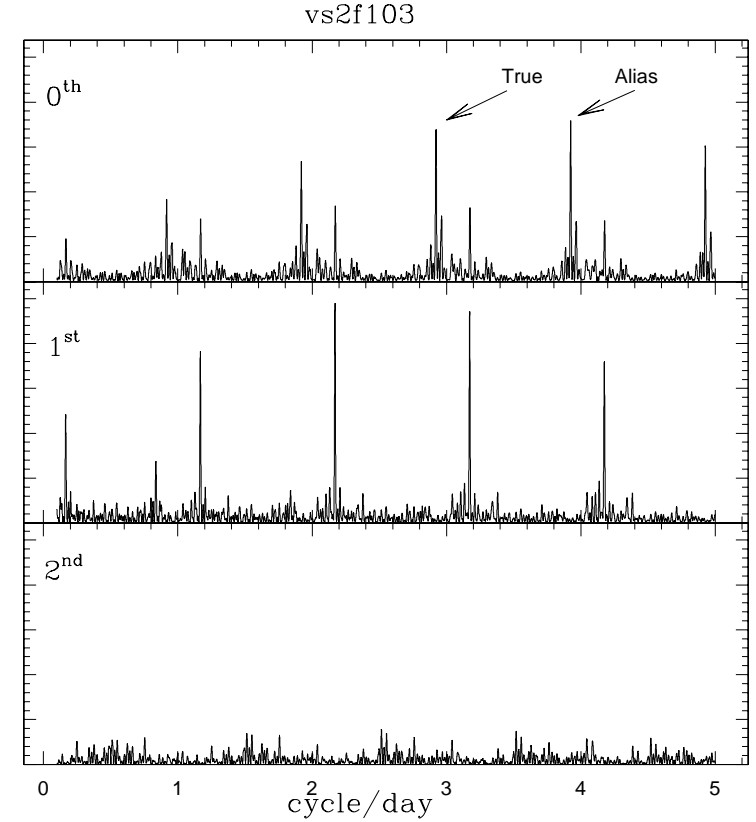

Fig. 2. Example of RRd star where the primary period is trapped in an alias period.

\subsection{Multi-periodic RR Lyraes}

\subsubsection{First method}

To search for multi-periodic RR Lyraes, we use the following procedure. For each lightcurve we search for the dominant period and fit a third order Fourier series to the folded lightcurve. The resulting Fourier series is then subtracted from the time series and the procedure is iterated. To characterize the strength of the peak value in the periodogram, we follow Alcock et al. (2000b) and calculate the statistics:

$S_{i}=\frac{A_{\mathrm{p}}-\langle A(\nu)\rangle}{\sigma_{A(\nu)}}$

where $A_{\mathrm{p}},\langle A(\nu)\rangle$ and $\sigma_{A(\nu)}$ are the peak value, average value and standard deviation of the periodograms respectively. $S_{i}$ corresponds to the $i$ th cycle. The procedure is iterated as long as $S_{i}>8$.

For all the lightcurves passing at least 2 iterations, we calculated the value $\chi_{\text {ratio }}=\chi_{1}^{2} / \chi_{2}^{2}$, where $\chi_{1}^{2}$ is the reduced $\chi^{2}$ of the fit of the Fourier series with the primary period and $\chi_{2}^{2}$ refers to the fit of the double Fourier series with the primary and secondary periods. All lightcurves for which $\operatorname{Proba}\left(\chi>\chi_{\text {ratio }}\right)<50 \%$ according to a FisherSnedecor distribution were selected for visual inspection. For all these candidates, we simultaneously checked the lightcurves and the periodograms in order to select the multi-mode pulsating stars.

\subsubsection{Second method}

It is very likely that we miss many multi-mode variables with the above-described procedure. This is due to three reasons: (1) the photometric accuracy is limited with our 
Table 1. Double mode RR Lyrae in Sgr. Amplitudes are given in $B_{\mathrm{J}}$ for $D U O$ and $B_{4415}$ for $S A G$.

\begin{tabular}{|c|c|c|c|c|c|c|c|c|c|c|}
\hline Ident. & $\alpha_{\mathrm{J} 2000}$ & $\delta_{\mathrm{J} 2000}$ & $B_{\mathrm{J}}$ & $B_{4415}$ & $A_{0}$ & $A_{1} / A_{0}$ & $P_{0}$ & $P_{1} / P_{0}$ & $P_{\mathrm{F}}$ & Method 1 \\
\hline vs $3 f 773$ & 183153.88 & -284907.1 & $\ldots$ & 19.1 & 0.16 & 2.33 & 0.60371 & 0.7447 & 0.00 & yes \\
\hline vs10f77 & 185324.68 & -294346.2 & $\ldots$ & 19.1 & 0.17 & 3.23 & 0.46500 & 0.7432 & 0.07 & yes \\
\hline vs4f75 & 185339.03 & -291905.9 & $\ldots$ & 19.0 & 0.29 & 1.83 & 0.55012 & 0.7466 & 0.06 & yes \\
\hline vs2f99 & 185226.75 & -282457.1 & $\ldots$ & 19.1 & 0.41 & 1.34 & 0.47776 & 0.7435 & 0.03 & yes \\
\hline vs $2 f 103$ & 185236.04 & $-29 \quad 1537.4$ & $\ldots$ & 19.3 & 0.46 & 1.15 & 0.46073 & 0.7425 & 0.06 & no (A1) \\
\hline vs7f161 & 185044.32 & -294152.1 & $\ldots$ & 19.3 & 0.31 & 1.74 & 0.46502 & 0.7426 & 0.06 & no (A01) \\
\hline vs1f242 & 184831.65 & -290015.1 & $\ldots$ & 19.2 & 0.39 & 1.49 & 0.52240 & 0.7460 & 0.01 & yes \\
\hline vs1f243 & 184806.14 & -291244.6 & $\ldots$ & 19.1 & 0.35 & 1.43 & 0.45935 & 0.7423 & 0.08 & yes \\
\hline vs17f249 & 184823.98 & -302514.0 & $\ldots$ & 19.1 & 0.41 & 1.29 & 0.47735 & 0.7440 & 0.02 & yes \\
\hline vs9f385 & 184329.01 & -294301.8 & $\ldots$ & 18.9 & 0.20 & 2.55 & 0.47961 & 0.7442 & 0.09 & yes \\
\hline vs $2 f 443$ & 184159.37 & -300623.6 & $\ldots$ & 19.1 & 0.33 & 1.54 & 0.46672 & 0.7432 & 0.02 & yes \\
\hline vs1f524 & 183949.07 & -292930.2 & $\ldots$ & 19.3 & 0.27 & 1.78 & 0.54114 & 0.7461 & 0.05 & yes \\
\hline vs14f598 & 183634.95 & -273640.6 & $\ldots$ & 19.7 & 0.35 & 1.49 & 0.54462 & 0.7456 & 0.07 & yes \\
\hline vs3f659 & 183453.71 & -283248.5 & $\ldots$ & 19.7 & 0.29 & 1.97 & 0.47647 & 0.7430 & 0.05 & yes \\
\hline vs8f105 & 185244.12 & -293924.1 & $\ldots$ & 19.1 & 0.16 & 3.19 & 0.47942 & 0.7439 & 0.20 & yes \\
\hline vs $2 f 266$ & 184744.99 & $-28 \quad 18 \quad 48.1$ & $\ldots$ & 19.6 & 0.21 & 2.38 & 0.46990 & 0.7436 & 0.11 & no $(\mathrm{A} 0)$ \\
\hline vs5f267 & 184723.95 & -282845.6 & $\cdots$ & 18.9 & 0.17 & 2.88 & 0.48187 & 0.7448 & 0.15 & yes \\
\hline vs12f466 & 184110.15 & -290442.5 & $\ldots$ & 19.1 & 0.16 & 3.00 & 0.47537 & 0.7432 & 0.15 & no $(\mathrm{A} 0)$ \\
\hline vs13f158 & 185036.45 & -290658.0 & $\ldots$ & 19.0 & 0.10 & 4.47 & 0.47258 & 0.7438 & 0.26 & yes \\
\hline vs3f170 & 184942.00 & -255657.1 & $\ldots$ & 20.2 & 0.25 & 2.70 & 0.48731 & 0.7455 & 0.29 & yes \\
\hline vs5f408 & 184308.95 & -284002.6 & $\ldots$ & 19.1 & 0.13 & 4.12 & 0.47129 & 0.7432 & 0.28 & no $(\mathrm{A} 0)$ \\
\hline vs5f468 & $1841 \quad 13.63$ & -293209.6 & $\ldots$ & 19.1 & 0.12 & 4.09 & 0.48087 & 0.7445 & 0.25 & no (A0) \\
\hline vs0f684 & 183444.91 & -275138.1 & $\ldots$ & 19.0 & 0.23 & 1.58 & 0.46836 & 0.7435 & 0.23 & no (A01) \\
\hline vs9f356 & 184453.06 & -293316.1 & $\ldots$ & 18.7 & 0.14 & 3.11 & 0.47159 & 0.7434 & 0.44 & no (A01) \\
\hline vs0f525 & 183953.39 & -293451.2 & $\ldots$ & 19.2 & 0.07 & 7.04 & 0.45021 & 0.7417 & 0.43 & no $(\mathrm{A} 0)$ \\
\hline $\mathrm{vd} 4 \mathrm{f} 61$ & 183114.89 & $-28 \quad 1451.3$ & 18.7 & $\ldots$ & 0.25 & 1.52 & 0.47203 & 0.7441 & 0.07 & yes \\
\hline vd1f63 & 183107.68 & -282954.2 & 18.1 & $\ldots$ & 0.19 & 2.74 & 0.47793 & 0.7440 & 0.08 & yes \\
\hline vd0f138 & 183000.81 & -321425.3 & 18.3 & $\ldots$ & 0.42 & 1.02 & 0.47218 & 0.7435 & 0.02 & yes \\
\hline vd1f176 & 182752.04 & -284627.1 & 18.3 & $\ldots$ & 0.32 & 1.31 & 0.49083 & 0.7443 & 0.09 & yes \\
\hline vd9f228 & 182557.57 & -280547.5 & 18.2 & $\ldots$ & 0.41 & 0.99 & 0.45725 & 0.7425 & 0.10 & no $(\mathrm{A} 0)$ \\
\hline $\operatorname{vd} 2 f 263$ & 182522.74 & -292441.7 & 18.3 & $\ldots$ & 0.42 & 1.34 & 0.47374 & 0.7434 & 0.02 & yes \\
\hline vd16f471 & 181833.38 & -313754.3 & 18.2 & $\ldots$ & 0.21 & 2.77 & 0.47716 & 0.7435 & 0.07 & yes \\
\hline vd4f101 & 183005.41 & -302410.0 & 18.0 & $\ldots$ & 0.13 & 4.16 & 0.47238 & 0.7432 & 0.16 & yes \\
\hline vd9f185 & 182748.51 & -303211.1 & 18.0 & $\ldots$ & 0.20 & 2.18 & 0.54381 & 0.7459 & 0.12 & yes \\
\hline vd14f208 & 182629.60 & -293752.0 & 18.2 & $\ldots$ & 0.43 & 1.46 & 0.46635 & 0.7433 & 0.14 & yes \\
\hline vd8f687 & 181129.88 & -300023.6 & 18.1 & $\ldots$ & 0.11 & 2.93 & 0.55626 & 0.7464 & 0.29 & yes \\
\hline vd1f141 & 184841.74 & -272438.6 & 17.6 & $\ldots$ & 0.30 & 1.91 & 0.47590 & 0.7443 & 0.03 & yes \\
\hline vd5f152 & 182843.59 & -293155.5 & 17.9 & $\ldots$ & 0.37 & 1.68 & 0.46495 & 0.7432 & 0.05 & yes \\
\hline vd11f353 & 182235.34 & -303152.3 & 17.9 & $\ldots$ & 0.25 & 1.47 & 0.47361 & 0.7441 & 0.13 & yes \\
\hline $\mathrm{vd} 21 \mathrm{f} 689$ & 181122.76 & -303312.7 & 17.6 & $\cdots$ & 0.09 & 4.38 & 0.51433 & 0.7453 & 0.25 & yes \\
\hline
\end{tabular}

Table 2. Same as Table 1 for Galactic RRd stars.

\begin{tabular}{lllllllllll}
\hline Ident. & $\alpha_{\mathrm{J} 2000}$ & $\delta_{\mathrm{J} 2000}$ & $B_{\mathrm{J}}$ & $B_{4415}$ & $A_{0}$ & $A_{1} / A_{0}$ & $P_{0}$ & $P_{1} / P_{0}$ & $P_{\mathrm{F}}$ & Method 1 \\
\hline & & & & & & & & & & \\
vs7f693 & 183356.78 & -293911.2 & $\ldots$ & 17.0 & 0.26 & 1.77 & 0.45626 & 0.7437 & 0.04 & yes \\
vs1f148 & 185106.00 & -271502.9 & $\ldots$ & 17.1 & 0.20 & 2.44 & 0.54103 & 0.7462 & 0.24 & yes \\
vs13f417 & 184237.13 & -302337.3 & $\ldots$ & 17.9 & 0.29 & 1.87 & 0.48769 & 0.7447 & 0.26 & no (A01) \\
vs14f440 & 184149.51 & -293139.7 & $\ldots$ & 16.0 & 0.11 & 5.15 & 0.48187 & 0.7447 & 0.32 & no (A0) \\
vs11f158 & 185046.72 & -290922.8 & $\ldots$ & 17.9 & 0.52 & 1.18 & 0.50367 & 0.7451 & 0.01 & yes \\
vs7f54 & 185446.05 & -303251.9 & $\ldots$ & 17.5 & 0.51 & 2.55 & 0.43574 & 0.7405 & 0.05 & yes \\
vd2f86 & 182954.97 & -273214.8 & 16.6 & $\ldots$ & 0.42 & 1.72 & 0.55026 & 0.7460 & 0.13 & yes \\
vd9f521 & 181654.04 & -302951.5 & 14.7 & $\ldots$ & 0.73 & 0.82 & 0.46157 & 0.7424 & 0.07 & no (A0) \\
vd21f652 & 181222.92 & -284934.4 & 16.8 & $\ldots$ & 0.40 & 1.36 & 0.47482 & 0.7434 & 0.06 & yes \\
vd15f747 & 180953.11 & -305056.3 & 16.6 & $\ldots$ & 0.42 & 1.49 & 0.47632 & 0.7444 & 0.02 & yes \\
vd20f737 & 180958.97 & -285225.5 & 16.3 & $\ldots$ & 0.18 & 2.05 & 0.57766 & 0.7456 & 0.30 & no (A0) \\
vd6f488 & 181807.38 & -293301.5 & 15.3 & $\ldots$ & 0.18 & 2.50 & 0.47560 & 0.7437 & 0.33 & no (N) \\
vd5f715 & 181109.59 & -300720.1 & 16.5 & $\ldots$ & 0.13 & 2.03 & 0.55489 & 0.7487 & 0.38 & no (N) \\
\hline
\end{tabular}


Table 3. Same as Table 1 for RR Lyrae stars in Sgr with two closely spaced frequencies. Subscripts p and s refer to the primary and secondary pulsations respectively.

\begin{tabular}{|c|c|c|c|c|c|c|c|c|c|c|}
\hline Ident. & $\alpha_{\mathrm{J} 2000}$ & $\delta_{\mathrm{J} 2000}$ & $B_{\mathrm{J}}$ & $B_{4415}$ & $A_{\mathrm{p}}$ & $A_{\mathrm{s}} / A_{\mathrm{p}}$ & $P_{\mathrm{p}}$ & $P_{\mathrm{s}} / P_{\mathrm{p}}$ & $P_{\mathrm{F}}$ & type \\
\hline vs $2 f 274$ & 184737.05 & -295050.7 & $\ldots$ & 19.0 & 0.28 & 0.71 & 0.29782 & 1.0283 & 0.01 & RRc \\
\hline vs4f335 & 184542.04 & -305234.3 & $\ldots$ & 19.0 & 0.33 & 0.39 & 0.26455 & 0.9601 & 0.21 & RRc \\
\hline vs4f391 & 184409.83 & -304831.5 & $\ldots$ & 19.3 & 0.26 & 0.75 & 0.29862 & 0.9710 & 0.05 & RRc \\
\hline vd8f264 & 182433.85 & -292829.4 & 18.2 & $\ldots$ & 0.31 & 0.68 & 0.26556 & 0.9981 & 0.06 & $\mathrm{RRc}$ \\
\hline vd8f661 & 181239.07 & -302813.9 & 18.9 & $\ldots$ & 0.36 & 0.47 & 0.26133 & 0.9770 & 0.17 & RRc \\
\hline
\end{tabular}

Table 4. Same as Table 3 for Galactic RR Lyraes.

\begin{tabular}{|c|c|c|c|c|c|c|c|c|c|c|}
\hline Ident. & $\alpha_{\mathrm{J} 2000}$ & $\delta_{\mathrm{J} 2000}$ & $B_{\mathrm{J}}$ & $B_{4415}$ & $A_{\mathrm{p}}$ & $A_{\mathrm{s}} / A_{\mathrm{p}}$ & $P_{\mathrm{p}}$ & $P_{\mathrm{s}} / P_{\mathrm{p}}$ & $P_{\mathrm{F}}$ & type \\
\hline vs4f114 & 185130.86 & -260418.7 & $\ldots$ & 18.2 & 0.32 & 0.28 & 0.22685 & 1.0074 & 0.35 & $\mathrm{RRc}$ \\
\hline vs3f146 & 185044.22 & -264939.2 & $\ldots$ & 18.1 & 0.47 & 0.45 & 0.26258 & 1.0151 & 0.00 & $\mathrm{RRc}$ \\
\hline vd1f77 & 183138.39 & -311914.2 & 16.4 & $\ldots$ & 0.35 & 0.52 & 0.29817 & 0.9778 & 0.05 & $\mathrm{RRc}$ \\
\hline vd25f234 & 182531.48 & -290717.1 & 13.9 & $\ldots$ & 0.33 & 0.67 & 0.26223 & 0.9824 & 0.18 & RRc \\
\hline $\mathrm{vd} 8 \mathrm{f} 324$ & 182302.84 & -301434.2 & 15.1 & $\ldots$ & 0.65 & 0.35 & 0.25743 & 1.0027 & 0.18 & $\mathrm{RRc}$ \\
\hline $\mathrm{vd} 25 \mathrm{f} 670$ & 181200.53 & -321351.9 & 15.2 & $\ldots$ & 0.44 & 0.59 & 0.28959 & 1.0193 & 0.12 & RRc \\
\hline vd8f704 & $1811 \quad 19.24$ & -275506.6 & 16.9 & $\ldots$ & 0.41 & 0.56 & 0.27036 & 0.9600 & 0.19 & $\mathrm{RRc}$ \\
\hline vd9f754 & 180944.70 & -320832.0 & 16.8 & $\ldots$ & 0.28 & 0.82 & 0.28066 & 1.0263 & 0.19 & $\mathrm{RRc}$ \\
\hline
\end{tabular}

photographic material, (2) the superposition of two (or more) periods introduces noise in the periodograms, (3) the strong aliasing at $\pm n$ day $^{-1}$ (see Fig. 1). There is not much that can be done about these problems, except for problem (3) in the search for RRd stars where we have more control because the period ratios are relatively well constrained. Figure 2 shows a typical example of a RRd star missed by the preceding method. Although the maximum amplitude occurs at $\nu_{\max }=3.9232 \mathrm{~d}^{-1}$, it is clear that the true frequency is rather $\nu_{0}=2.9232 \mathrm{~d}^{-1}$, a value that yields a more "classical" period ratio $\nu_{0} / \nu_{1}=0.7425$.

We thus re-processed the whole variable star data-base in order to search for double-mode RR Lyrae stars, but this time we forced the period search between $0.33^{\mathrm{d}}$ and $0.44^{\mathrm{d}}$. This interval encompasses the range of primary periods of all known RRds. A Fourier series with the periodicity $P_{1}$ was fitted to the light curve and the residual relative to this fit was period searched in the range $0.45^{\mathrm{d}}<P_{0}<0.60^{\mathrm{d}}$. The fit to the residuals was then subtracted from the initial light curve and the procedure was repeated. We then calculated the reduced $\chi^{2}$ about the resulting double Fourier series.

In the following, we kept only those stars for which $0.74 \leqslant P_{1} / P_{0} \leqslant 0.75$, corresponding to a range that is considered physically plausible by stellar pulsation models (Cox et al. 1980; Kovács et al. 1991; Bono et al. 1996). To select the double mode pulsating RR Lyraes we define the variable $\chi_{\text {ratio }}=\chi_{1}^{2} / \chi_{2}^{2}$, where $\chi_{1}^{2}$ and $\chi_{2}^{2}$ are the reduced $\chi^{2}$ referring to the single-mode and double-mode fit respectively. All the variables for which the double-mode fit yields a better representation of the light curve (i.e. those with Proba $\left(\chi>\chi_{\text {ratio }}\right)<50 \%$ ) where inspected visually. We simultaneously checked the single period and the double-mode fit in order not to spuriously select a variable with a true period outside the range searched as an RRd star.

For completeness, we repeated the above-described procedure by inverting the order of the period searches $\left(P_{0}\right.$ before $\left.P_{1}\right)$ in order to detect any eventual RRd with a higher amplitude in the fundamental mode relative to the first overtone mode. No additionnal RRd star was found this way, confirming that the first overtone mode is almost always the primary pulsation. This method allowed detection of 16 additional RRd stars.

\subsubsection{Results}

The final sample contains two kinds of multiperiodic stars: RRd stars and RR Lyrae stars with two closely spaced frequencies. No attempt was made to detect RR Lyrae variables implying long time effects (e.g. Blazkho variables or period changes), because of our too short time span for these kinds of detections. Nor did we search for more than two periods because of the limited accuracy of our photometry.

We found 53 RRd stars (40 in Sgr and 13 in the Galaxy), which are presented in Tables 1 and 2. The first column is the star identifier, Cols. 2 and 3 are the equatorial coordinates. The apparent magnitude of each star is shown in Col. $4(D U O)$ or $5(S A G)$. Columns 6 and 7 are the amplitude (in $B_{\mathrm{J}}$ band for $D U O$ and $B_{4415}$ for $S A G$ ) of the fundamental mode pulsation, and amplitude ratio respectively. Columns 8 and 9 are the periods and period ratios. Column 10 represents the probability that the $\chi_{\text {ratio }}$ occured by chance if it were following a FisherSnedecor distribution. Although this is not true because of the non-Gaussianity of the errors, it is still indicative of the strength of the detection. In the last column, we 

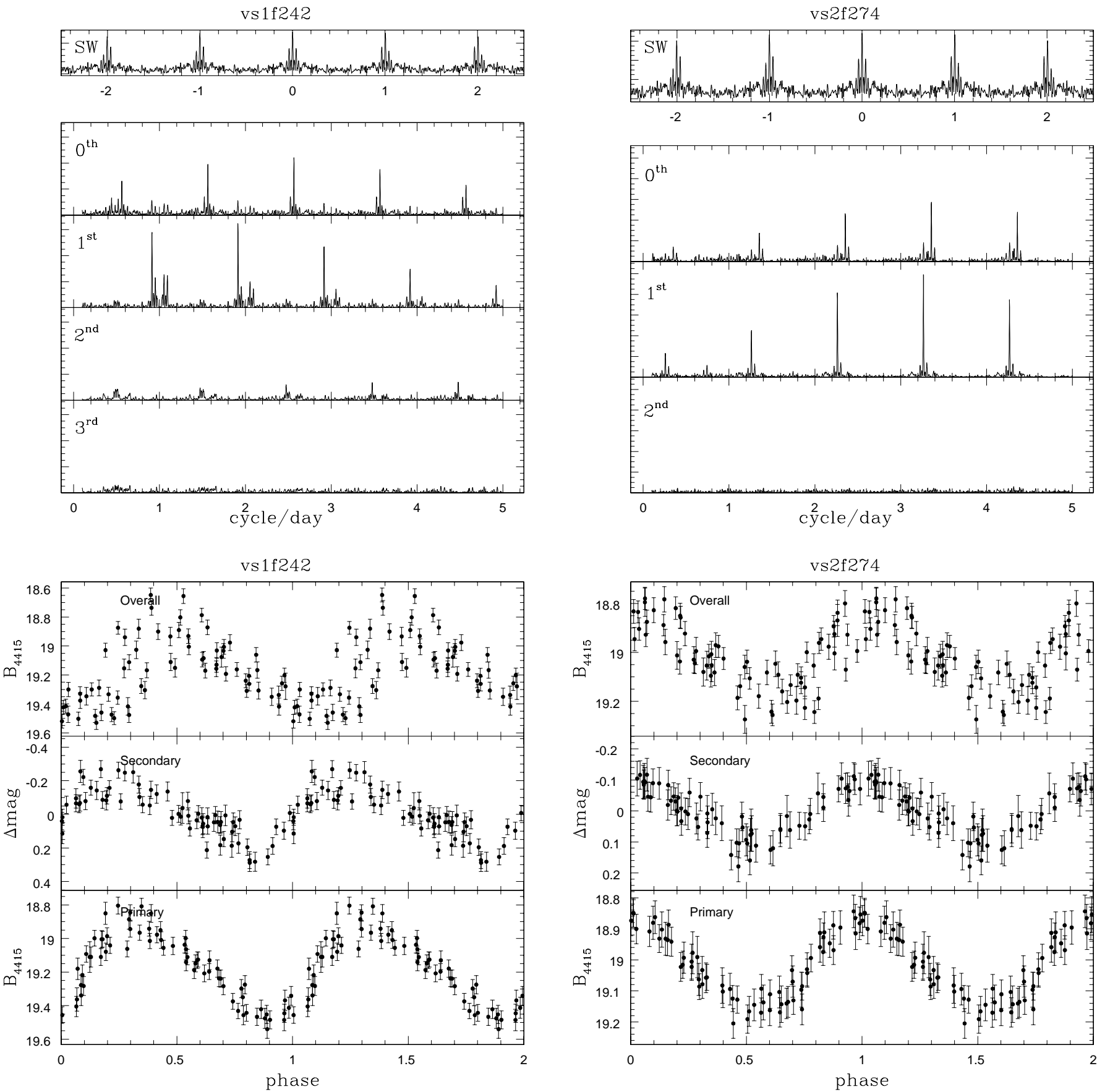

Fig. 3. Left panels: a typical RRd star. The panels represent from top to bottom: spectral window, periodogram after successive subtraction of the main pulsation, and decomposition of the lightcurve. Right panels: same as before for a RR Lyrae star with two closely spaced frequencies.

indicate whether the RRd star has been detected through the first method or not, and in the latter case, why it was missed ( $A 0$ : fundamental period aliased, $A 1$ : first overtone period aliased, $N$ : periodogram too noisy - i.e. $S_{i}<8$ ). A typical RRd star is presented in Fig. 3 (left panels).

In addition to the RRd stars, we found $13 \mathrm{RR}$ Lyrae stars with two closely spaced frequencies (5 in Sgr and 8 in the Galaxy). These stars are presented in Table 3 (Sgr) and 4 (Galaxy). A typical detection is presented in Fig. 3 (right panels). Similar detections have been performed in M 55 (Olech et al. 1999a), M 5 (Olech et al. 1999b), the LMC (Alcock et al. 2000b) and the Galactic Bulge (Moskalik 2000).

Finding charts of all the detected multiperiodic RR Lyraes are presented in Fig. 4 only available in electronic form at http://www.edpsciences.org. North is up, East is left, and each box is $36^{\prime \prime}$ on a side. Light curves are available at the CDS via anonymous ftp to cdsarc.u-strasbg.fr $(130.79 .128 .5)$ or via http: // cdsweb.u-strasbg.fr/cgi-bin/qcat?J/A+A/ $375 / 909$. 


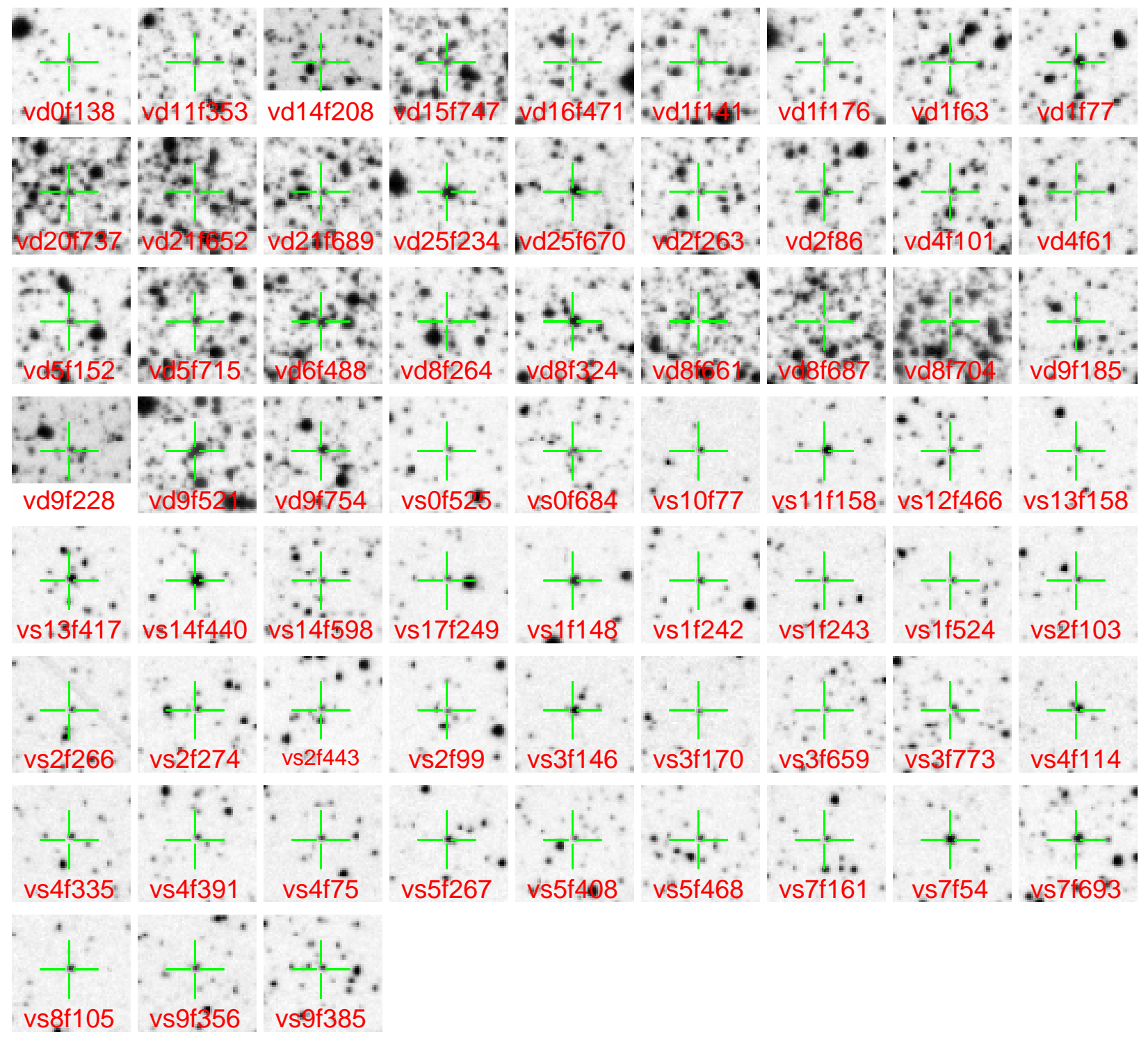

Fig. 4. Finding charts for multiperiodic RR Lyrae stars in Sgr and the Galactic Centre. Each finding chart is $36^{\prime \prime}$ on a side. North is oriented up and East is left.

\section{Period distributions}

\subsection{Single-mode RR Lyrae stars}

The mean periods of $\mathrm{RR}$ Lyrae stars in Sgr are $0.574^{\mathrm{d}} \pm 0.002$ and $0.322^{\mathrm{d}} \pm 0.002$ for RRab and RRc stars respectively. This can be compared to the mean periods found in OoI $\left(0.55^{\mathrm{d}}\right.$ and $\left.0.32^{\mathrm{d}}\right)$ and OoII $\left(0.65^{\mathrm{d}}\right.$ and $\left.0.37^{\mathrm{d}}\right)$ groups. Sgr is thus to be classified in the OoI group. This is not common for Dsph galaxies, which are usually located in the intermediate region of the Oosterhoff dichotomy. Table 6 presents the mean periods of RR Lyrae stars in an OoI type globular cluster (M 3), an OoII type cluster (M 15), in the Galactic Centre and in all the Milky Way satellites with a well-studied RR Lyrae population. The LMC RR Lyrae stars have been extracted from photographic plate time series of the first EROS season whereas sources for all the other systems are listed in the caption of Table 6 .

The RR Lyrae stars in Sgr present the shortest average periods among all the dwarf galaxies, but slightly longer than the OoI globular cluster M 3 and the Galactic Centre. Indeed, these values are rather close to those found in the Large Magellanic Cloud $\left(\left\langle P_{\mathrm{ab}}\right\rangle=0.582^{\mathrm{d}} \pm 0.001\right.$ and $\left.\left\langle P_{c}\right\rangle=0.325^{\mathrm{d}} \pm 0.001\right)$.

The similarity between Sgr and the LMC is even more striking when one considers the period distributions of the RR Lyraes. The period distributions of RR Lyrae stars in the Galactic Centre and in different dwarf galaxies are shown in Fig. 5 and are compared to that of Sgr in Fig. 6. The coefficients of correlation between all these histograms and the period distribution in Sgr are shown in Col. 7 of 
Table 6. Mean periods of RR Lyrae stars in dwarf galaxies of the local group. RR Lyrae data are taken from (Draco) Nemec (1985), (Ursa Minor) Nemec et al. (1988), (Carina) Saha et al. (1986), (Leo II) Siegel \& Majewski (2000), (Sculptor) Kałużny et al. (1995), (Sextans) Mateo et al. (1995), (SMC) Smith et al. (1992) and Graham (1975), Caretta et al. (1998), Silbermann \& Smith (1995).

\begin{tabular}{lccccccc}
\hline System & {$[\mathrm{Fe} / \mathrm{H}]$} & $\left\langle P_{\mathrm{ab}}\right\rangle$ & $\sigma_{\mathrm{ab}}$ & $\left\langle P_{\mathrm{c}}\right\rangle$ & $\sigma_{\mathrm{c}}$ & $r$ & $p_{\mathrm{s}}$ \\
\hline M 3 & -1.7 & 0.558 & 0.008 & 0.344 & 0.019 & 0.87 & -24.0 \\
M 15 & -2.2 & 0.641 & 0.013 & 0.359 & 0.008 & 0.15 & -1068.5 \\
Draco & -2.0 & 0.614 & 0.004 & 0.351 & 0.012 & 0.67 & -48.9 \\
Ursa Minor & -2.2 & 0.638 & 0.009 & 0.375 & 0.011 & 0.16 & -63.0 \\
Carina & -2.0 & 0.620 & 0.006 & 0.366 & 0.015 & 0.51 & -60.8 \\
Leo II & -1.9 & 0.619 & 0.006 & 0.363 & 0.008 & 0.62 & -42.9 \\
Sculptor & -1.8 & 0.587 & 0.007 & 0.337 & 0.005 & 0.67 & -46.7 \\
Sextans & -1.7 & 0.606 & 0.010 & 0.355 & 0.024 & 0.78 & -44.6 \\
SMC (RRLyr) & -1.7 & 0.588 & 0.006 & 0.380 & 0.008 & 0.81 & -34.5 \\
LMC (RRLyr) & -1.6 & 0.582 & 0.001 & 0.325 & 0.001 & 0.96 & -19.0 \\
Sgr & -1.6 & 0.574 & 0.002 & 0.322 & 0.002 & 1.00 & $\ldots$ \\
Galactic Centre & $\ldots$ & 0.548 & 0.002 & 0.305 & 0.002 & 0.86 & -28.3 \\
\hline
\end{tabular}

Table 6. As expected, the highest correlation is reached for the $\mathrm{LMC}^{3}$ where $r=0.96$.

Since the correlation coefficient $r$ measures the linearity between two distributions, it is sensitive to the shape of the distributions, but not to shifts (i.e. two identical distributions shifted one with respect to the other would have $r=1.00)$. We thus performed another test, adapted from maximum likelihood statistics, and which is more sensitive to shifts. First, each period histogram is normalized to that of Sgr. We then calculate the values $p_{\mathrm{s}}=\Sigma_{i} \log p_{i}$ where $p_{i}$ corresponds to the probability of finding the number of stars observed in the $i$ th bin if the parent distribution was identical to the period histogram of Sgr. The results are shown in Col. 8 of Table 6 . The highest $p_{\mathrm{s}}$ value is reached by the $\mathrm{LMC}$, confirming that the period distributions in Sgr and the LMC are the most similar.

The RR Lyrae samples in Sgr and the LMC are large ( $\sim 1700$ in Sgr and $\sim 6500$ in the LMC), making the resemblance between these two systems significant. This resemblance is even more striking when one considers that no two other dwarf galaxies have similar period distributions. Note however that the two distributions are not identical, this being excluded at the $99 \%$ level by a KS test.

\subsection{Double-mode RR Lyrae stars}

Table 7 summarizes the average periods of RRd stars in all the systems known to harbor this kind of variable. Again, the LMC and Sgr show a remarkable similarity in the mean periods of their RRd star population. In Fig. 7 we present the distributions of all the published RRd stars in the Petersen diagram. The cluster RRd variables and

\footnotetext{
${ }^{3}$ For the correlation between Sgr and the LMC we excluded the bins $P \in\left[0.32^{\mathrm{d}}, 0.34^{\mathrm{d}}\right]$ and $P \in\left[0.48^{\mathrm{d}}, 0.50^{\mathrm{d}}\right]$ because of poor phase coverage for the EROS stars in these period ranges caused by the nearly daily sampling of the light curves.
}

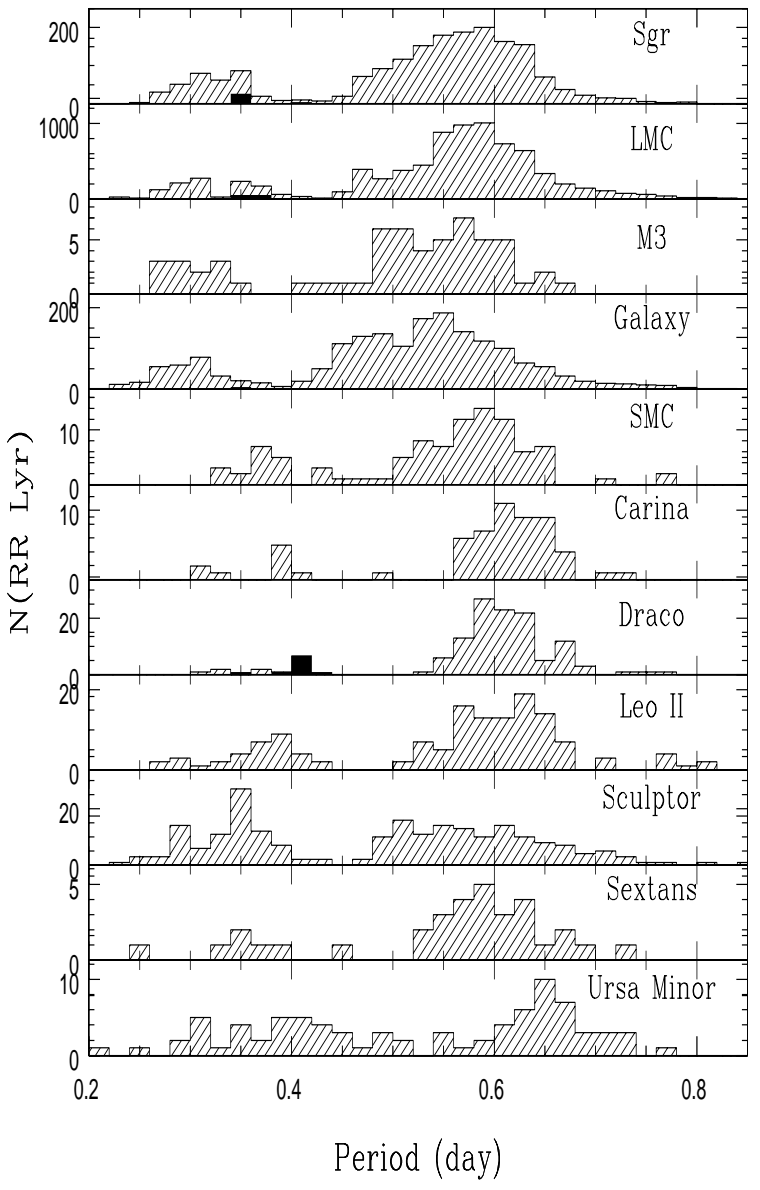

Fig. 5. Period distribution of the RR Lyraes detected in various systems. The dark regions indicate the location of the RRd variables (1 st overtone period).

those of the Draco Dsph galaxy occupy two distinct regions, reproducing the Oosterhoff dichotomy. This is no more the case for RRd stars in Sgr, in the LMC and in the Galactic Centre. These latter stars are distributed on a strip across the diagram. These distributions can be explained either by a metallicity spread, a mass spread or a combination of both (Kovács 2000). A metallicity spread in the RRd population of the LMC has already been confirmed by spectroscopic measurements (Clementini et al. 2000; Bragaglia et al. 2001) and it is likely that the same applies to Sgr.

To compare further, we transformed the Petersen diagrams into density maps and calculated the correlation between all these distributions. The results are shown in Col. 6 of Table 7. The correlation between the LMC and Sgr is $r=0.77$, which is not as good as the correlation between the single-mode RR Lyrae period distributions, but differences in the completeness between samples may affect the comparison.

\section{Period-amplitude diagram}

The period-amplitude diagram is presented in Fig. 8a. To construct this figure, we considered only the $S A G$ field, 

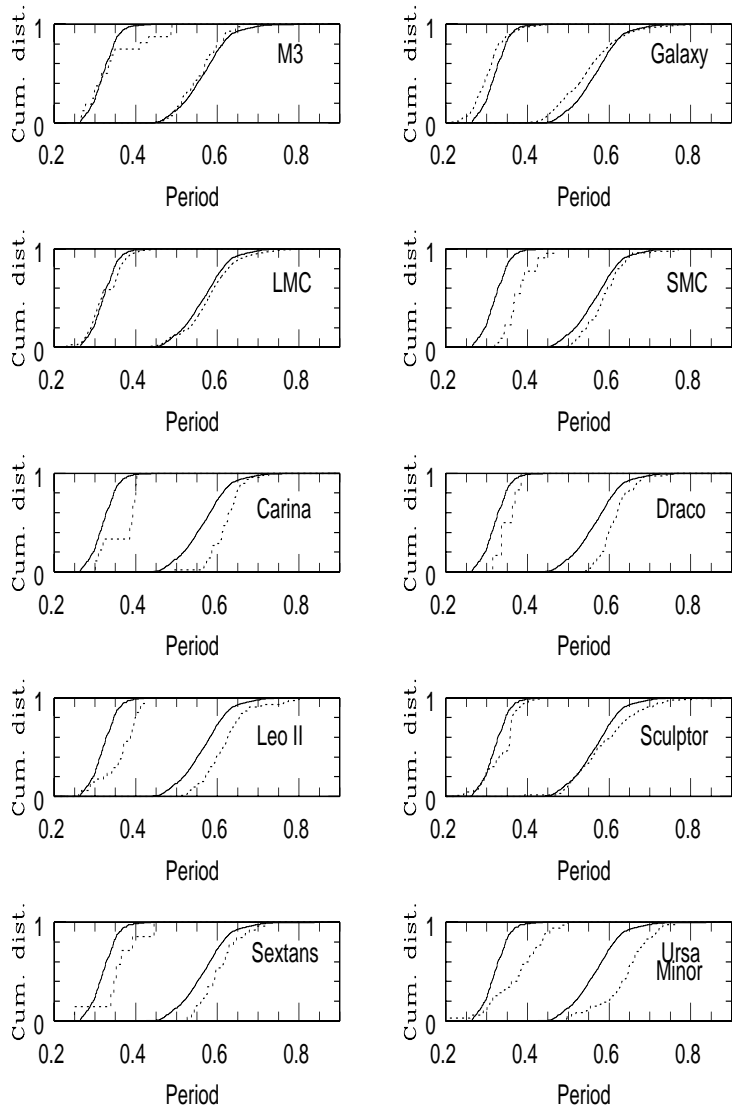

Fig. 6. Cumulative distributions of RR Lyrae periods in the OoI globular cluster M 3 and all satellites of the Milky Way with a well studied RR Lyrae population. The solid line refers to Sgr whereas the dotted line corresponds to the system indicated in each panel. Left (resp. right) curves correspond to RRc stars (resp. RRab stars).

which has a more precise photometry than DUO, resulting in a higher accuracy of the amplitudes. As will be shown in Sect. 7, the RR Lyrae population is homogeneous over the field and there is therefore no loss of generality by restricting ourself to $S A G$. We rescaled the amplitudes in $S A G$ to the $V$ amplitude from the relation $A_{V}=0.93( \pm 0.17) A_{4415}-0.05( \pm 0.16)$, inferred from 15 RR Lyrae stars in common with those detected by Layden \& Sarajedini (2000) near M 54.

The distribution of RRab stars in Fig. 8a presents a high dispersion but is preferentially clumped around the ridge line of the globular cluster M 3, confirming that this population is related to the OoI group. However, the distribution is skewed toward the ridge line of the OoII cluster member M 9, suggesting the presence of a metal-poor subpopulation within the RR Lyrae population. The RRab population is dominated by low-amplitude stars with a ratio $N\left(A_{V}>1.0 \mathrm{mag}\right) / N\left(A_{V}<1.0 \mathrm{mag}\right)=0.55$. The $\mathrm{RR}$ Lyrae population is thus dominated by RRb stars, indicative of a red horizontal branch morphology.

Remarkably, the same features are apparent in the period-amplitude diagram of the LMC (Alcock et al. 1996;

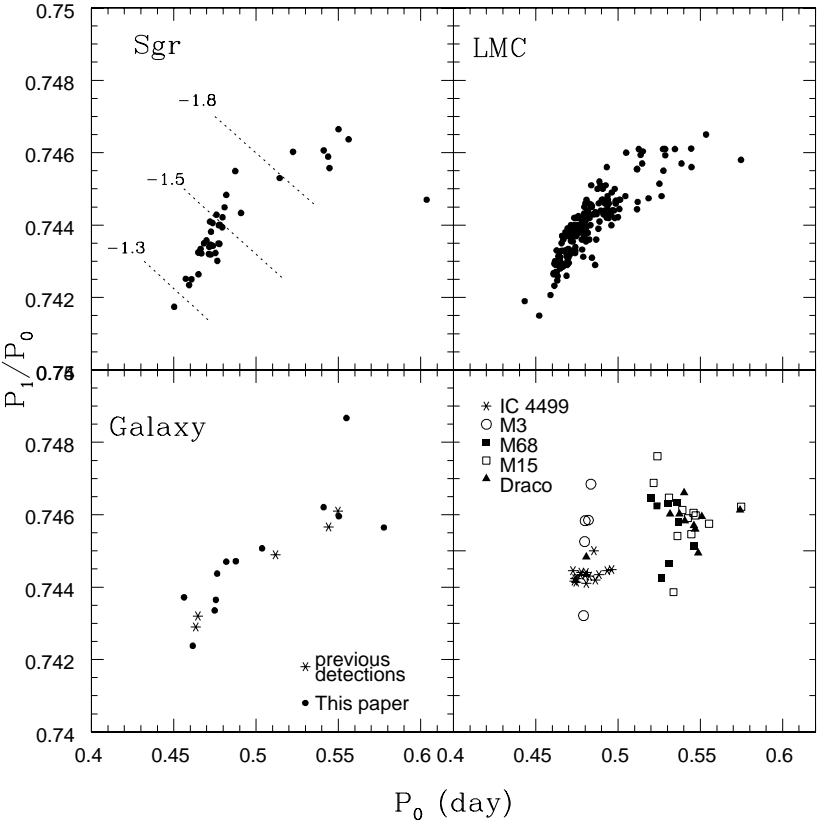

Fig. 7. Petersen diagram for all the published RRd. In the upper left panel, the dotted lines correspond to the metallicity for a specific model of RRd star (see text). From top to bottom $[\mathrm{Fe} / \mathrm{H}]=-1.8,-1.5$ and -1.3 dex. In the lower left panel, asterisks are RRd stars presented in the literature whereas heavy dots correspond to RRd stars presented in this paper.

Table 7. Summary of RRd stars detected to date. Metallicities are taken from Mateo (1998) for dwarf galaxies and from Harris (1996) for globular clusters. Informations on RRd stars are taken from IC 4499: Walker \& Nemec (1996), M 3: Nemec \& Clement (1989), Corwin et al. (1999), NGC 2419 and NGC 6426: Clement \& Nemec (1990), M 68: Clement et al. (1993), M 15: Nemec (1985), Draco Dsph: Nemec (1985), Sculptor: Kałużny et al. (1995), LMC: Alcock et al. (2000b), MW: Clement et al. (1991), Garcia-Melendo \& Clement (1997), Clementini et al. (2000)

\begin{tabular}{lccccc}
\hline System & {$[\mathrm{Fe} / \mathrm{H}]$} & $N(\mathrm{RRd})$ & $\left\langle P_{0}^{\mathrm{RR}_{\mathrm{d}}}\right\rangle$ & $\left\langle P_{1}^{\mathrm{RR}}{ }_{\mathrm{d}}\right\rangle$ & $\mathrm{r}$ \\
\hline IC 4499 & -1.5 & 17 & 0.481 & 0.358 & 0.44 \\
M 3 & -1.7 & 5 & 0.481 & 0.359 & 0.22 \\
NGC 2419 & -2.1 & 1 & 0.546 & 0.407 & $\ldots$ \\
M 68 & -2.1 & 8 & 0.531 & 0.396 & -0.02 \\
M 15 & -2.2 & 12 & 0.541 & 0.404 & 0.13 \\
NGC 6426 & -2.2 & 1 & 0.542 & 0.404 & $\ldots$ \\
Draco Dsph & -2.0 & 10 & 0.540 & 0.403 & 0.15 \\
Sculptor & -1.8 & 1 & $?$ & 0.404 & $\ldots$ \\
LMC & -1.7 & 181 & 0.485 & 0.361 & 0.77 \\
Sgr & -1.1 & 40 & 0.487 & 0.362 & 1.00 \\
MW & $\ldots$ & 18 & 0.504 & 0.376 & 0.54 \\
\hline
\end{tabular}

Alcock et al. 2000a), providing new evidence of the similarity of the RR Lyrae populations between these two galaxies. 

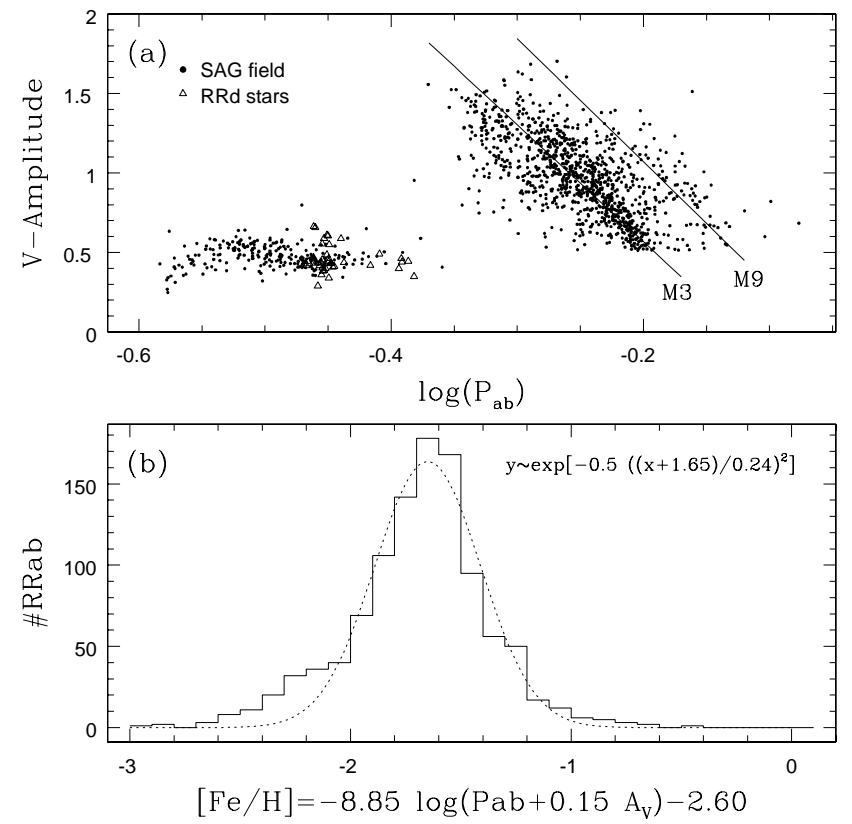

Fig. 8. Panel a) period/amplitude diagram of the RR Lyrae stars in Sgr. Dots represent stars detected in $S A G$ and triangle are double-mode RR Lyraes. Also shown are the fiducial lines of M 3 (OoI group) and M 9 (OoII group). Panel b) Metallicity estimate from the period-amplitude-metallicity relation. The dotted line represents the best Gaussian fit, whose relation is given in the upper right corner of the panel.

\section{Metallicity estimate of the RR Lyrae population}

\subsection{Period-Metallicity relations}

Some indication on the metallicity of the RR Lyrae population in Sgr can be inferred from its period distribution. In a study of cluster and field RR Lyrae stars spanning a wide range of metallicities, Sandage (1993) related the average periods of RR Lyrae stars to their metallicities:

$$
\begin{aligned}
& {[\mathrm{Fe} / \mathrm{H}]_{\mathrm{ZW}}=\left(-\log \left\langle P_{\mathrm{ab}}\right\rangle-0.389\right) / 0.092} \\
& {[\mathrm{Fe} / \mathrm{H}]_{\mathrm{ZW}}=\left(-\log \left\langle P_{\mathrm{c}}\right\rangle-0.670\right) / 0.119}
\end{aligned}
$$

where $\left\langle P_{\mathrm{ab}}\right\rangle$ and $\left\langle P_{\mathrm{c}}\right\rangle$ are the average periods of RRab and RRc stars respectively. Although these relations were derived from cluster and field RR Lyraes, Siegel \& Majewski (2000, their Fig. 6) showed that RR Lyrae populations in Dsph followed the same relation. Applying Eqs. (2) and (3) to Sgr yields an average metallicity of $[\mathrm{Fe} / \mathrm{H}]=-1.61$ and -1.49 dex respectively.

The location of the blue and red fundamental edge of the instability strip are functions of metallicity. The shortest and longest period RR Lyraes are thus indicative of the metallicity boundaries of the RR Lyrae population which are given as (Sandage 1993):

$$
\begin{aligned}
& {[\mathrm{Fe} / \mathrm{H}]_{\mathrm{ZW}}{ }^{(\max )}=\left[-\log \left(P_{\mathrm{ab}}^{\min }\right)-0.500\right] / 0.122-0.2} \\
& {[\mathrm{Fe} / \mathrm{H}]_{\mathrm{ZW}}{ }^{(\min )}=\left[-\log \left(P_{\mathrm{ab}}^{\max }\right)-0.280\right] / 0.090-0.2}
\end{aligned}
$$

where $P_{\mathrm{ab}}^{\min }$ and $P_{\mathrm{ab}}^{\max }$ are the minimum and maximum periods of RRab stars respectively. We added the constant terms in Eqs. (4) and (5) in order to rescale the metallicity to the Zinn \& West (1984) scale, whose zero point is $\sim 0.2$ dex more metal-poor than the Butler \& Blanco metallicity scale (Blanco 1992) on which these equations are based. The shortest RRab period is $0.41531^{\mathrm{d}}$, yielding an upper limit of $[\mathrm{Fe} / \mathrm{H}]=-1.17$ dex, whereas the longest period of $0.84400^{\mathrm{d}}$ implies a lower limit of $[\mathrm{Fe} / \mathrm{H}]=-2.49$ dex. These values suggest a considerable spread in the metallicity of the RR Lyrae population.

\subsection{Period-amplitude-metallicity relation}

Alcock et al. (2000a) provide a period-amplitudemetallicity relation calibrated with high-quality $V$-band light curves of RR Lyrae stars in several globular clusters:

$[\mathrm{Fe} / \mathrm{H}]=-8.85 \log \left(P_{\mathrm{ab}}+0.15 A_{V}\right)-2.60$

where $A_{V}$ is the amplitude in the $V$-band. The resulting metallicity distribution of Sgr RRab member stars is presented in Fig. 8b. The peak-value is at $[\mathrm{Fe} / \mathrm{H}] \simeq$ -1.65 dex, close to the estimate inferred from the Sandage relations. This is also similar to the value found in the $\mathrm{LMC}$ with the same relation $([\mathrm{Fe} / \mathrm{H}] \simeq-1.6$; Alcock et al. 2000a).

The best Gaussian fit to the metallicity distribution is given by $y \propto \mathrm{e}^{-\frac{1}{2}\left(\frac{x+1.65}{0.24}\right)^{2}}$. As can be seen in Fig. 8b, this function fits the distribution relatively well, except for $[\mathrm{Fe} / \mathrm{H}] \lesssim-2.0$ dex where the presence of a significant metal-poor subpopulation is apparent.

\subsection{Petersen diagram}

The strong metallicity spread in the RR Lyrae population seems confirmed by the distribution of RRd stars in the Petersen diagram, although a dispersion in the star masses could also be responsible for the spread. The dotted lines in Fig. 7 represent metallicities for a specific model of RRd with a mass of $0.75 M_{\odot}$, a luminosity $\log \left(L / L_{\odot}\right)=1.72$, a hydrogen abundance $X=0.76$ and an effective temperature of 6900,6800 and $6700 \mathrm{~K}$ for $[\mathrm{Fe} / \mathrm{H}]=1.3,-1.5$ and -1.8 dex respectively. This model has been taken from Kovács (2000). The positions of the RRd stars are in good agreement with the above estimate of mean metallicity and metallicity spread of the RR Lyrae population.

A clump of RRd stars is apparent in Fig. 7 at $[\mathrm{Fe} / \mathrm{H}]<$ -1.8 dex, suggesting the presence of a minor but significant population of low metallicity and/or high mass RR Lyraes. The loci of the long period RRd stars in the Petersen diagram is similar to those found in OoII systems. Table 7 summarizes all the systems with known RRd pulsators. One sees that qs all these OoII systems have a metallicity within -2.0 and -2.2 dex, supporting the assumption that a fraction of RR Lyrae stars in Sgr has this abundance.

The existence of a very low metallicity population in Sgr has been suggested by Bellazzini et al. (1999a, 1999b) who detected a star count excess in a region of 

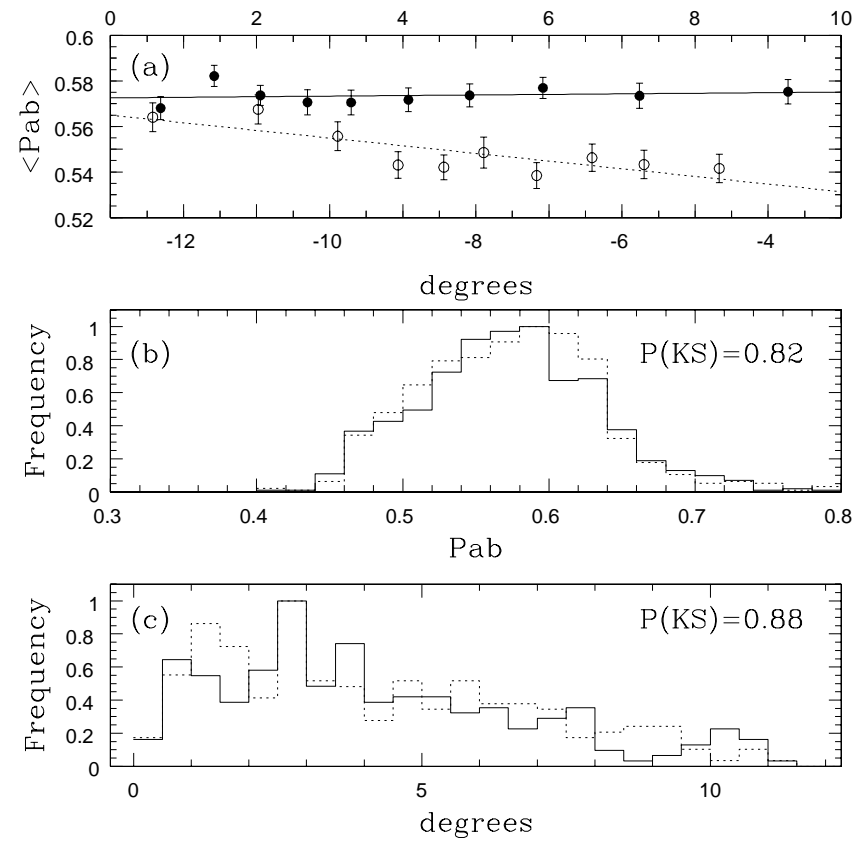

Fig. 9. Panel a) average period of RRab stars as a function of position. Filled circles correspond to RRab variables in Sgr and a distance $(X)$ from M 54 projected onto the main axis (upper labels). Open circles are average periods of Galactic RRab and are plotted as a function of Galactic latitude (lower labels). Panel b) Normalized period histograms of RRab stars. The solid line correspond to RRab stars with $X<5^{\circ}$ whereas the dotted line correspond to RRabs with $X>5^{\circ}$. Panel c) Normalized histograms of $X$ for the 250 shortest period (solid line) and longest period (dotted line) RRab variables in Sgr.

the CMD that could represent a very blue horizontal branch. Furthermore, in a period-amplitude diagram of RR Lyrae stars towards the globular cluster M 54, Layden \& Sarajedini (2000) noted that a fraction of RR Lyrae stars could be consistent with a contamination by Sgr field stars of metallicity $[\mathrm{Fe} / \mathrm{H}]=-2.1$ dex.

\section{Homogeneity of the RR Lyrae population over the field}

Several authors claim having detected a metallicity gradient in the stellar population of Sgr, the centre being more metal-rich than the outer regions. This finding is based on the redder morphology of the Red Giant Branch and the Horizontal Branch at the centre of Sgr relative to the outer regions (Marconi et al. 1998; Bellazzini et al. 1999b; Alard 2001). If confirmed, this feature would be in concordance with observations of other dwarf galaxies (e.g. Saviane et al. 2001 and references therein). We now question whether such a gradient is apparent in the RR Lyrae population.

Any metallicity gradient would translate into a gradient in the mean period of RRab stars. Figure 9a presents the mean period of RRab stars in Sgr (solid line) as a function of distance from M54 projected onto the main axis $^{4}$. Each bin size has been adapted in order to contain the same number of RRab stars. A linear least square fit through these points yields a slope of $\partial\left(\left\langle P_{\mathrm{ab}}\right\rangle\right) / \partial X=$ $0.0002( \pm 0.0004)$. From Eq. (2), it results in a metallicity difference $<0.1$ dex between the two extremities of the field. Clearly, there is no significant metallicity gradient in the RR Lyrae population of Sgr. For comparison, we present in Fig. 9a the average period of Galactic RRab as a function of latitude (dotted line), where the period dependence as a function of position is evident.

To test the homogeneity further, we divided the RRab catalogue into two subsamples around the median distance from M 54 and compared the period distributions (Fig. 9b). A KS test shows that the two histograms are drawn from the same parent distribution with a probability of $82 \%$, suggesting a similar horizontal branch morphology in the two subsamples.

Finally, in Fig. 9c we compare the spatial distributions of the 250 longest and shortest period RRab variables. The KS test yields a probability of $88 \%$ for the two histograms to be issued from the same parent distribution. This definitely excludes any modification of the period distribution with position. Thus, unless a metallicity gradient and the second parameter effect conspire to keep the horizontal branch morphology constant over the field, the RR Lyrae population should be homogeneous in the main body of Sgr. Furthermore, if the metallicity gradient was confirmed, this may indicate that the RR Lyrae population is not associated to the prominent red horizontal branch which is apparent in the CMDs of Sgr.

\section{Estimation of the number of RR Lyrae stars in Sgr}

Using the results from Paper I, it is possible to estimate the total number of RRab stars in Sgr. The best fit to the core of Sgr corresponds to an exponential with a scalelength of $4.1^{\circ}$ on the main axis and a central density of 139 RRab per square degree. Assuming an axis ratio of 3:1 (Ibata et al. 1995), we get $\sim 4200$ RRab in the main body of Sgr. This estimate is only a lower limit for Sgr since we did not consider the nearly flat profile in the outer region of the main body (Paper I). Irwin (1999) estimated that Sgr lost $50 \%$ of its mass through tidal stripping. This implies that the number of RRab stars associated with Sgr could be as high as $\sim 8400$.

Provided RRab stars are good tracers of light in Sgr, we can estimate a lower limit to the total luminosity of this galaxy using the luminosity function (LF) published by Mateo et al. (1995). Their LF is complete down to $M_{V} \sim 4.5$ mag. For fainter magnitudes we extend the LF with the Bulge LF (Holtzman et al. 1998). This should not significantly alter the result since most of the light is emitted by brighter stars. The field of Mateo et al. contains five RRab stars. Rescaling the RRab number density to the integrated luminosity and assuming a distance modulus of

\footnotetext{
${ }^{4}$ We assume a position angle of $108.6^{\circ}$ for Sgr.
} 


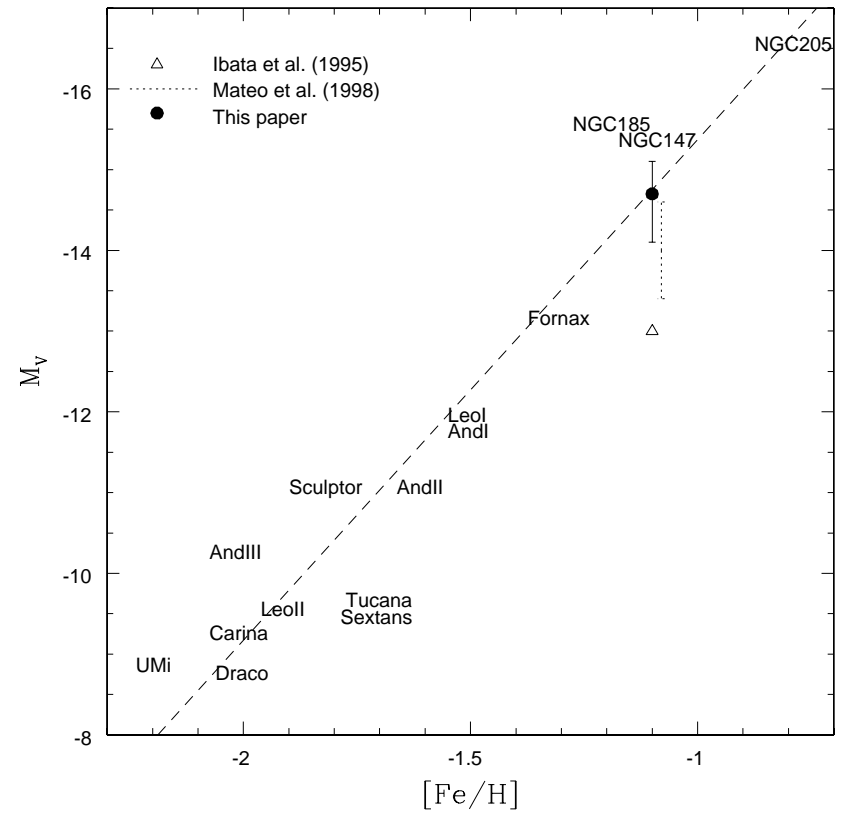

Fig. 10. Luminosity-metallicity relation for Dsph of the Local group. Data have been taken from Mateo (1998). The dashed line corresponds to the best fit to the data points. The filled circle corresponds to the estimated luminosity of Sgr if this galaxy had lost $50 \%$ of its mass since its formation. The open triangle represents the first luminosity estimate of this galaxy (Ibata et al. 1995). The vertical dotted line (shifted by 0.02 dex for clarity) encompasses the different estimates of Mateo et al. (1998).

$(m-M)_{0}=17.0$ to Sgr yields $-4.9 \mathrm{mag}$ per RRab. It results that $M_{V}(\mathrm{Sgr})<-13.9_{-0.6}^{+0.4} \mathrm{mag}$, where the uncertainties represent the poissonian uncertainty in the RRab counts. Again, if we assume $50 \%$ mass loss, the total magnitude of Sgr before stripping would be $\sim-14.7$ mag.

Previous estimates have continuously increased the luminosity of Sgr as new extensions of this galaxy were discovered, with estimations ranging from $M_{V} \sim-13.0$ (Ibata et al. 1995) to $M_{V} \sim-14.6 \mathrm{mag}$ (Mateo et al. 1998). Our estimation seems to favor the higher luminosity of Sgr. The metallicity of the dominant population in Sgr is estimated to be $[\mathrm{Fe} / \mathrm{H}] \sim-1.1$ dex (Mateo et al. 1995). An absolute luminosity of $\sim-14.7$ mag for Sgr would be consistent with the empirical magnitude-metallicity relationship for Dsph galaxies, as shown in Fig. 10.

\section{Summary and conclusion}

We summarize our results below:

1. The average period of RR Lyrae stars in Sgr places this galaxy in the long period tail of the Oosterhoff I group;

2. We found 53 double-mode RR Lyraes (40 in Sgr and 13 in the Galaxy), and 13 RR Lyrae stars with two closely spaced pulsation frequencies ( 5 in $\operatorname{Sgr}$ and 8 in the Galaxy). The multi-periodic RR Lyraes in Sgr are the first such stars ever discovered in this galaxy whereas the 13 foreground RRds increase the number of known Galactic RRd stars to 18;

3. The period and amplitude distributions of the RR Lyrae population in Sgr suggest an average metallicity of $\sim-1.6$ dex, with a contribution of a minor but perceptible population at $[\mathrm{Fe} / \mathrm{H}] \lesssim-2.0$ dex. Furthermore, together with the period distribution of RRd stars, there seems to be a large metallicity spread within the RR Lyrae population;

4. We find no significant variation of the period distribution over our field, suggesting a homogeneous population. There is therefore no evidence of a metallicity gradient in the RR Lyrae population. This result is in contradiction with claims of a metallicity gradient within the main body of Sgr, unless the RR Lyrae population is not directly related to the other populations;

5. We find a striking similarity between the RR Lyrae populations in Sgr and the LMC. This similarity is based on comparisons of the period distributions, the period-amplitude diagram, and, to a lesser extent, the period distributions of RRd stars;

6. We estimate the total number of RRab stars to be $\sim 4200$ in the main body of Sgr. If Sgr has lost $50 \%$ of its mass since its formation, the total number of RRab stars associated with Sgr would be $\sim 8400$. If RRab stars trace light, the above estimates would correspond to an integrated $V$-magnitude of $-13.9 \mathrm{mag}$ for the main body, and -14.7 mag for the whole system.

The stellar population of Sgr has a high metallicity dispersion. This was first suggested from interpretation of the Color-Magnitude diagram of Sgr (Marconi et al. 1998; Bellazzini et al. 1999a) and later confirmed by spectroscopic observations with values ranging from -1.4 dex up to solar abundance (Smecker-Hane \& McWilliam 1999; Bonifacio 2000). It seems that the RR Lyrae population also presents a high dispersion of $\sim 1$ dex around $[\mathrm{Fe} / \mathrm{H}] \simeq-1.6$, suggesting that the early star-formation process in Sgr was complex. Sgr could thus have formed on a large scale with star bursts occuring in several gaseous clumps of different metallicities. Another scenario would be a continuous star formation with progressive metal enrichment.

The similarity of RR Lyrae populations between Sgr and the LMC is indicative of similar horizontal branch morphologies. This similarity implies that the parameter(s) driving the HB morphology are similar in both systems. Furthermore, since RR Lyraes represent the old metal-weak population of these systems, this result suggests that the LMC and Sgr formed at the same epoch and in a similar environment with respect to the metal abundance. It is thus tempting to speculate that Sgr and the LMC had a common progenitor. In this picture, Sgr could correspond to a piece of the LMC pulled out during a collision with the Galaxy. Such a scenario could help to explain how a galaxy with an old stellar population can be observed on such a low orbit without being completely disrupted through Galactic tides. 
Acknowledgements. I am pleased to thank Christophe Alard for constant support and many useful suggestions during the elaboration of this paper.

\section{References}

Alard, C. 1996, ApJL, 458, 17

Alard, C., \& Guibert, J. 1997, A\&A, 326, 1

Alard, C. 2001, in preparation

Alcock, C., Allsman, R., Alves, D., et al. (MACHO collaboration) 1996, AJ, 111, 1146

Alcock, C., Allsman, R., Alves, D., et al. (MACHO collaboration) 1997, ApJ, 482, 89

Alcock C., Allsman, R., Alves, D., et al. (MACHO collaboration) 2000a, AJ, 119, 2194

Alcock, C., Allsman, R., Alves, D., et al. (MACHO collaboration) 2000b, ApJ, 542, 257

Bellazzini, M., Ferraro, F., \& Buonanno, R. 1999a, MNRAS, 304, 633

Bellazzini, M., Ferraro, F., \& Buonanno, R. 1999b, MNRAS, 307, 619

Benkö, J., \& Jurcsik, J. 2000, ASP, 203, 257

Blair, M., \& Gilmore, G. 1982, PASP, 94, 742

Blanco, V. 1992, AJ, 104, 734

Bonifacio, P. 2000, ESO Proc., ed. J. Bergeron, \& A. Renzini, 381

Bono, G., Caputo, F., Castellani, V. \& Marconi, M. 1996, ApJL, 471, 33

Bragaglia, A., Gratton, R., Carretta, E., et al. 2001 [astro-ph/0103515]

Caretta, E., Cacciari, C., Ferraro, F. R., Fusi Pecci, F., \& Tessicini, G. 1998, MNRAS, 298, 1005

Clement, C., \& Nemec, J. 1990, JRASC, 84, 434

Clement, C., \& Walker, I. 1991, AJ, 101, 1352

Clement, C., Kinman, T., \& Suntzeff, N. 1991, ApJ, 372, 273

Clement, C., Ferance, S., \& Simon, N. 1993, ApJ, 412, 183

Clement, C., Hilditch, R., Kałużny, J., \& Rucinski, S. 1997, ApJL, 489, 55

Clementini, G., Di Tomaso, S., Di Fabrizio, L., et al. 2000, AJ, 120,2054

Clementini, G., Bragaglia, A., \& Di Fabrizio, L., et al. 2000, ASP Conf. Ser., 203, 172

Corwin, M., Carney, B., \& Allen, D. 1999, AJ, 117, 1332

Cox, A., King, D., \& Hodson, S. 1980, ApJ, 236, 219

Cseresnjes, P., Alard, C., \& Guibert, 2000, A\&A, 357, 871, Paper I

Dziembowski, W., \& Cassisi, S. 1999, AcA, 49, 371

Garcia-Melendo, E., \& Clement, C. 1997, AJ, 114, 1190

Graham, J. 1975, PASP, 87, 641

Harris, W. E. 1996, AJ, 112, 407

Holtzman, J., Watson, A., Baum, W., et al. 1998, AJ, 115, 1946

Ibata, R., Gilmore, G., \& Irwin, M. 1995, MNRAS, 277, 781

Irwin, M. 1999, Stellar Content of the Local Group, IAU 192, ed. P. Whitelock, \& R. D. Cannon, San Fransisco: Astron. Soc. Pac., 409
Jerzykiewicz, M., \& Wenzel, W. 1977, AcA, 27, 35

Kałużny, J., Kubiak, M., Szymański, M., et al. 1995, A\&AS, 112, 407

Kałużny, J., Hilditch, R., Clement, C., \& Rucinski, S. 1998, MNRAS, 296, 347

Kovács, G., Buchler, R., \& Marom, M. 1991, A\&A, 252, L27

Kovács, G., \& Walker, A. 1999, ApJ, 512, 271

Kovács, G. 2000, Nonlinear Studies of Stellar Pulsation, ed. M. Takeuti, \& D. Sasselov, Astrophysics and Space Science Library Series (Kluwer), in press

Layden, A., \& Sarajedini, A. 2000, AJ, 119, 1760

Mateo, M., Udalski, A., Szymański, M., et al. 1995, AJ, 109, 588

Mateo, M., Fischer, P., \& Krzemiński, M. 1995, AJ, 110, 2166

Mateo, M. 1998, ARA\&A, 435

Mateo, M., Olszewski, E., \& Morrison, H. 1998, ApJL, 55

Marconi, G., Buonanno, R., Castellani, M., et al. 1998, A\&A, 330, 453

Moskalik, P. 2000, The impact of large-Scale surveys on Pulsating Star Research, Conf. Ser., 203, 315

Nemec, J. 1985, AJ, 90, 204

Nemec, J. 1985, AJ, 90, 240

Nemec, J., Nemec, A., \& Norris, J. 1986, AJ, 92, 358

Nemec, J., Wehlau, A., \& De Oliveira, C. M. 1988, AJ, 96, 528

Nemec, J., \& Clement, C. 1989, AJ, 98, 860

Olech, A., Kałużny, J., Thompson, I. B., et al. 1999a, AJ, 118, 442

Olech, A., Woźniak, P. R., Alard, C., et al. 1999b, MNRAS, 310,759

Oosterhoff, P. 1939, Observatory, 62, 104

Paparó, M., Saad, S. M., Szeidl, B., Abu Elazm, M. S., \& Sharaf, M. A. 1998, A\&A, 332, 102

Parker, Q., \& Malin, D. 1999, Publ. Astron. Soc. Aust., 16, 288

Petersen, J. 1973, A\&A, 27, 89

Phillipps, S., \& Parker, Q. 1993, MNRAS, 265, 385

Purdue, P., Silbermann, N., Gay, P., \& Smith, H. 1995, AJ, 110,1712

Saha, A., Monet, D., \& Seitzer, P. 1986, AJ, 92, 302

Sandage, A. 1982, ApJ, 282, 553

Sandage, A. 1993, AJ, 106, 687

Sarajedini, A., \& Layden, A. 1995, AJ, 109, 1086

Saviane, I., Held, E., Momany, Y., \& Rizzi, L. 2001 [astro-ph/0101250]

Schwarzenberg-Czerny, A. 1996, ApJL, 460, 107

Siegel, M., \& Majewski, S. 2000, AJ, 120, 284

Silbermann, N., \& Smith, H. 1995, AJ, 110, 704

Smecker-Hane, T., \& Mc William, A. 1999, ASP Conf. Ser., 192, 150

Smith, H., Silbermann, N., Baird, S., \& Graham, J. 1992, AJ, 104,1430

Van Hoolst, T., Dziembowski, W., \& Kawaler, S. D. 1998, MNRAS, 297, 536

Walker, A., \& Nemec, J. 1996, AJ, 112, 2026

Zinn, R., \& West, M. 1984, ApJS, 55, 45 